\title{
Inverse Vector Function Hilbert Boundary Value Problem
}

\author{
Ding Yun, Yang Xiaochun* \\ Department of Mathematics, Dalian Maritime University, Dalian, P. R. China \\ Email address: \\ dingyun741121@hotmail.com (Ding Yun), yangxiaochun@dlmu.edu.cn (Yang Xiaochun) \\ *Corresponding author
}

\section{To cite this article:}

Ding Yun, Yang Xiaochun. Inverse Vector Function Hilbert Boundary Value Problem. Pure and Applied Mathematics Journal. Vol. 5, No. 5, 2016, pp. 160-164. doi: 10.11648/j.pamj.20160505.14

Received: August 28, 2016; Accepted: September 9, 2016; Published: September 28, 2016

\begin{abstract}
In the paper, how to solve an irregular vector function Hilbert boundary value inverse problem is discussed in generalization. In the solving, some diagonal matrices are introduced for helping to regulate those equations of Hilbert boundary value problem. Then, the solution of the problem is given.
\end{abstract}

Keywords: Vector Function, Irregular, Inverse Problem, Hilbert Boundary Value Problem, General Solution

\section{Introduction}

Hilbert Boundary Value problem or H- problem is a single boundary value problem. It has a strong relative to the Riemann boundary value problem or R-problem, which is a double boundary value problem.

For regular or irregular H-problem, even regular vector function $\mathrm{H}$-problem, we can find that there are a lots of benefit works and results. References [1-5] are some classical treatises in the region, in which many important results were collected. What need to be pointed out is that, in recently twenty years, famous scholar Lu JianKe and his group have done a lot of work in this region, especially on the periodic R-problem and double periodic R-problem. Some of these results were collected in the reference of book [1], and Refs [5-9] which related to this paper directly. It was pity to say that most of these works were written in Chinese, which affects the international communication in the region more or less.

Though the results about Riemann boundary value problem are obtained richly, there is few works on vector function R-problem [8] or H-problem [9], much less than that of irregular Riemann boundary value problem. How to solve those kind of problems? Refs [10] gave some constructive methods to solve a regular vector R-Problem. But the solving process was only given by description. There was no practice formula in the whole solving process and no analysis expression of solution in the results.

In the research of the vector function Riemann boundary value problem, solving the problem need to use the matrix theory because of the coefficient matrix in the problem is a function matrix whose elements are functions). As we know, there is no good method to deal with this kind matrix. One of the most important tool to deal with matrix is determinant. Since the operation between determinant and matrix is quite different, and there are many isolated zero and singular points on the matrix, which made the solving of the problem is very difficult. Furthermore, solving the problem must reduce a basic solution matrix (or basic solution groups) to a canonical matrix (or canonical solution matrix). This means an effective method should be used to clean up all isolated zero and singular points from the basic solution matrix.

To overcome the difficulty of the partial index problem in solving [1-3], paper [11] put forward an effective method. That is, by introducing some diagonal matrices, transforms the original solving problem to an equivalent problem that the solving only need to determine the total index of the matrix but the partial index. Furthermore, papers [11] also gave a general method to deal with the problem that the determinant of a matrix equals to zero, which greatly helped to solve the regular vector function R-Problem or $\mathrm{H}$ - problem, even irregular of those problems.

Riemann boundary value inverse problem has been put forward for no more than twenty years. Nevertheless Riemann boundary value inverse problem (inverse R-problem) was normally named by Lu Jianke et al and was last supported by national natural of science funds of China (three times). Of cause, the new mention in Lu et al overcome some defects on logical that implied in the past research works [10-11]. 
As the irregular vector function inverse $\mathrm{H}$-problem is a new course, any new result hasn't been seen by far.

In this paper, a vector function Riemann boundary value inverse problem is discussed. In the solving, we fully apply the character and difference of the coefficient matrix. And by introducing a diagonal matrix, transfer the irregular vector function inverse $\mathrm{H}$ - problem to an equivalent regular vector function inverse $\mathrm{H}$-problem. Then by using the result in paper [8-9] and [11], the general solution of the problem is obtained.

In addition, we put more effort in simplifying the form of the solution. A Hermite interpolation polynomial is constructed which greatly reduce the order of the polynomial appearing in the solution. This means the final result is much better in using.

\section{Illustration}

The paper studies irregular vector function Riemann boundary value inverse problem. For the sample of, we still supposes curve $L$ is smoothness, $\in H$, and its positive direction is anticlockwise. Noted $D^{+}$as the curve's interior, $D^{-}$as the curve's exterior, and $0 \in D^{+}$. Notes

$$
\phi(z)=\left(\begin{array}{c}
\phi_{1}(z) \\
\phi_{2}(z) \\
\vdots \\
\phi_{n}(z)
\end{array}\right)=\left(\phi_{1}(z), \phi_{2}(z), \cdots, \phi_{2}(z)\right)^{\mathrm{T}} .
$$

Mention of the problem Irregular vector function $\mathrm{H}$-problem is that finding double vector functions $(\Phi(z), H(t))$ as form (1) with jumps on L, satisfying

$$
\begin{array}{r}
\operatorname{Re}\left\{\left[a_{1}(t)+i b_{1}(t)\right] \Phi^{+}(t)\right\}=c_{1}(t) H(t), t \in L \\
\operatorname{Re}\left\{\left[a_{2}(t)+i b_{2}(t)\right] \Phi^{+}(t)\right\}=c_{2}(t) H(t), t \in L
\end{array}
$$

in here, $L$ is direct supposed as a unit circle: $|t|=1, \Phi^{+}(t)$ is positive boundary of an unknown sectional holomorphic vector function, $\in H$ on $L$, and the singular order of $\Phi(z)$ at $\infty$ is finite. $H(t)$ is an unknown vector function, $\in H$ on L. $a_{l}(t), b_{l}(t)(l=1,2)$ and $c_{l}(t)$ are all $n \times n$ matrices in which all elements are real functions and $\in H$ on $L$. Without losing the generality, we just solve the problem in the class $R_{0}$.

Compared with the regular Riemann boundary value problem, det $g_{t}(t)(t=1,2)$ could have several zero point on L, else $\operatorname{det}\left[g_{2}(t)-g_{1}(t)\right]$ and $\operatorname{det}\left[g_{2}(t) G_{1}(t)-g_{1}(t) G_{2}(t)\right]$. For simplifying, it still supposes $\operatorname{det} g_{t}(t) \neq 0(\mathrm{t}=1,2)$, and notes

$$
\begin{gathered}
G_{1}(t)=-\left[a_{1}(t)+i b_{1}(t)\right]^{-1}\left[a_{1}(t)-i b_{1}(t)\right],(l=1,2) \\
\mathrm{g}_{1}(t)=-\left[a_{1}(t)+i b_{1}(t)\right]^{-1} \mathrm{c}_{1}(t) .(l=1,2)
\end{gathered}
$$

define on $|t|>1$, that is,

$$
\Phi_{*}(z)=\overline{\Phi\left(\frac{1 / \bar{z}}{z}\right)}
$$

We get a sectional holomorphic vector function

$$
\Phi(z)=\left\{\begin{array}{ll}
\Phi(z) & |z|<1 \\
\Phi_{*}(z) & |z|>1
\end{array} .\right.
$$

and

$$
\Phi^{-}(t)=\Phi_{*}^{-}(t)=\overline{\Phi^{+}(t)} .
$$

Expression (2), (3) is changed to inverse R-problem [5]:

$$
\begin{gathered}
\Phi^{+}(t)=G_{1}(t) \Phi^{-}(t)+g_{1}(t) H(t), t \in L \\
\Phi^{+}(t)=G_{2}(t) \Phi^{-}(t)+g_{2}(t) H(t), t \in L
\end{gathered}
$$

here $G_{l}(t)=\left(G_{j k}^{l}(t)\right)_{n \times n}(l=1,2)$, given by (4), a known $n \times n$ matrix, its elements $\in H$ on $L ; g_{l}(t)=\left(g_{j k}{ }^{l}(t)\right)_{n \times n}(l$ $=1,2$ ) given by (5), else a known $n \times n$ matrix, its elements $\epsilon$ $H$ on $L$.

Since all $\operatorname{det} g_{l}(t) \quad(l=1,2), \quad \operatorname{det}\left[g_{2}(t)-g_{1}(t)\right]$, $\operatorname{det}\left[g_{2}(t) G_{1}(t)-g_{1}(t) G_{2}(t)\right]$ could have some zeroes on L, problem (9),(10) is an irregular inverse R-problem.

Similar to the discussion of the regular R-problem, if we make the same systematical prolongation as (6) on $\Phi(z)$ after it would find, and let

$$
\Omega(z)=\frac{1}{2}\left[\Phi(z)+\Phi_{*}(z)\right]
$$

then the condition (8) will be satisfied automatically. This is to say that $\Omega(z)$ is the solution of irregular inverse R-problem $[1,8]$. Hence, the next step is to obtain the $\Phi(z)$.

Before solving the problem, we introduce some notes here.

Suppose the zero points of $\operatorname{det}\left[g_{2}(t)-g_{1}(t)\right]$ on $L$ are $\sigma_{1}, \sigma_{2}, \cdots \cdots, \sigma_{l}$, and the order of them are $\varepsilon_{1}, \varepsilon_{2}, \cdots \varepsilon_{l}$, separately. Noted

$$
K(t)=\prod_{s=1}^{l}\left(t-\sigma_{s}\right)^{\varepsilon_{j}},
$$

and let $T(t)=(T(t))_{n \times n}$ a diagonal matrix, the elements on diagonal are $T_{k k}(t)$, and $T_{k k}(t)=1(k=1, \cdots, n-1)$, $T_{n n}(t)=1 / K(t) ; T^{*}(t)=\left(T^{*}(t)\right)_{n \times n}$ is another diagonal matrix, and its elements on diagonal are $T_{k k}{ }^{*}(t), T_{k k}{ }^{*}(t)$ $=1(k=1, \cdots, n-1)$, but $T_{n n}{ }^{*}(t)=K(t)$. 


\section{Solving the Problem}

Left multiplied a function matrix $g_{2}(t)$ to the two sides of (1.2), and left multiplied a function matrix $g_{1}(t)$ to the two sides of (4), (5) too, then plus the two expressions, it has

$$
\left[g_{2}(t)-g_{1}(t)\right] \Phi^{+}(t)=\left[g_{2}(t) G_{1}(t)-g_{1}(t) G_{2}(t)\right] \Phi^{-}(t)
$$

For solving the problem, first of all, we left multiplied a function matrix $T(t)$ on (12), it is easy to prove that $\operatorname{det}\left\{T(t)\left[g_{2}(t)-g_{1}(t)\right]\right\} \neq 0$ now.

Continuously, left multiplied a function matrix $\left\{T(t)\left[g_{2}(t)-g_{1}(t)\right]\right\}^{-1}$ on the two sides, we have

$$
\Phi^{+}(t)=\tilde{G}(t) \Phi^{-}(t)
$$

here,

$$
\tilde{G}(t)=\left\{T(t)\left[g_{2}(t)-g_{1}(t)\right]\right\}^{-1} T(t)\left[g_{2}(t) G_{1}(t)-g_{1}(t) G_{2}(t)\right] .
$$

Suppose the zero points of $\operatorname{det} \tilde{G}(t)$ and $1 / \operatorname{det} \tilde{G}(t)$ on $L$ are $\alpha_{1}, \alpha_{2}, \cdots \cdots, \alpha_{p}$ and $\beta_{1}, \beta_{2}, \cdots \cdots, \beta_{q} \quad\left(\alpha_{j} \neq \beta_{k}\right)$ respectively.

Their order are $\lambda_{1}, \lambda_{2}, \cdots \cdots, \lambda_{p}$ and $\mu_{1}, \mu_{2}, \cdots \cdots, \mu_{q}$, noted

$$
\begin{gathered}
\Pi_{1}(t)=\prod_{j=1}^{p}\left(t-\alpha_{j}\right)^{\lambda_{j}}, \Pi_{2}(t)=\prod_{k=1}^{q}\left(t-\beta_{k}\right)^{\mu_{k}}, \\
\Pi(t)=\Pi_{1}(t) / \Pi_{2}(t) \\
\lambda=\sum_{j=1}^{p} \lambda_{j}, \mu=\sum_{k=1}^{q} \mu_{k}
\end{gathered}
$$

$D(t)=(D(t))_{n \times n}$ is a diagonal matrix too, which elements on diagonal are $d_{k k}(t),\left(d_{k k}(t)=1 \quad(k=1, \cdots, n-1)\right.$, but $\left.d_{n n}(t)=1 / \Pi(t)\right) ; D^{*}(t)=\left(D^{*}(t)\right)_{n \times n}$ else a diagonal matrix, which elements on diagonal are $d_{k k}{ }^{*}(t)\left(d_{k k}{ }^{*}(t)\right.$ $=1(k=1, \cdots, n-1)$, but $\left.d_{n n}{ }^{*}(t)=\Pi(t)\right)$. Let

$$
G_{0}(t)=D^{*}(t) \tilde{G}(t),
$$

then

$$
\operatorname{det} G_{0}(t) \neq 0,1 / \operatorname{det} G_{0}(t) \neq 0 \text {. }
$$

Obviously, problem (13) is transferred to

$$
D_{1}(t) \Phi^{+}(t)=G_{0}(t) D_{2}(t) \Phi^{-}(t),
$$

where $D_{1}(t), D_{2}(t)$ are diagonal matrices whose construct is just like matrix $D(t)$. The only difference is that elements on diagonal now are replaced by $\Pi_{2}(t)$ and $\Pi_{1}(t)$ respectively.

Introduce a new sectional holomorphic vector function:

$$
\Psi(z)=\left\{\begin{array}{ll}
D_{2}(z) \Phi(z) & z \in D^{+} \\
D_{1}(z) \Phi(z) & z \in D^{-}
\end{array},\right.
$$

then, the problem (13) is transferred to

$$
\Psi^{+}(t)=G_{0}(t) \Psi^{-}(t) .
$$

It is a regular vector function $\mathrm{R}$ - problem whose canonical solution matrix can be found in refs [1].

Of course, we can simple use the results in the book [1] and write the solution just the same as there. This is, notes the canonical solution matrix is $X(z)$, so,

$$
X^{+}(t)=G_{0}(t) X^{-}(t), \text { or } G_{0}(t)=X^{+}(t)\left[X^{-}(t)\right]^{-1}
$$

furthermore

$$
\left[X^{+}(t)\right]^{-1} \Psi^{+}(t)=\left[X^{-}(t)\right]^{-1} \Psi^{-}(t) .
$$

Since we suppose the order of $\Phi(z)$ at $\infty$ is finite, by the Liouville theorem, the solution of problem (12) in $R_{0}$ is

$$
\Phi(z)=\left\{\begin{array}{l}
D_{2}^{*}(z)\left(P_{\kappa+\lambda}(z) \cdot X(z)\right) \\
D_{1}^{*}(z)\left(P_{\kappa+\lambda}(z) \cdot X(z)\right)
\end{array}=\left\{\begin{array}{l}
D_{2}^{*}(z) \sum_{k=1}^{n} P_{k}(z) X^{k}(z) \\
D_{1}^{*}(z) \sum_{k=1}^{n} P_{k}(z) X^{k}(z)
\end{array}\right.\right.
$$

where, $\kappa$ is the total index of matrix $G_{0}(t)^{[1-3]} ; D_{1}^{*}(t)$, $D_{2}{ }^{*}(t)$ are diagonal matrices whose construction is the same as the matrix $D(t)$, its elements on diagonal now are replaced by $1 / \Pi_{2}(t)$ and $1 / \Pi_{1}(t)$ respectively; $X(z)=$ $\left(X^{1}(z), X^{n}(z), \cdots \cdots, X^{n}(z)\right) \quad, \quad P_{\kappa+\lambda}(z)=$ $\left(P_{1}(z), P_{2}(z), \cdots \cdots, P_{n}(z)\right)$, all $P_{k}(z)(k=1, \ldots, n-1)$ are polynomials whose order is no more then $\kappa+\lambda$. Because the vector function of $\Phi^{+}(t), \Phi^{-}(t)$ should be bounded on $\mathrm{L}$, the last term in polynomial vector $P_{\kappa+\lambda}(z)$ must be taken as

$$
P_{n}(z)=D_{1}(t) D_{2}(t) P_{\kappa-\mu}(z) .
$$

That is

$$
\Phi(z)=\left\{\begin{array}{l}
D_{2}^{*}(z)\left(P_{\kappa+\lambda}(z) \cdot X(z)\right) \\
D_{1}^{*}(z)\left(P_{\kappa+\lambda}(z) \cdot X(z)\right)
\end{array} .\right.
$$

This method can be extended to the class $R_{m}$, especially in $R_{-1}$, and the result will be easily obtained. In fact, the only difference is that the order of polynomial vector here is not equal to $\kappa+\lambda$.

Afterward, taking $\Phi(z)$ into (2), (3) or (4), (5), then, we can get $H(t)$ :

$$
H(t)=g_{1}^{-1}(t)\left\{D_{2}^{*}(t)\left(P_{\kappa+\lambda}(t) \cdot X(t)\right)-G_{1}(t) D_{1}^{*}(t)\left(P_{\kappa+\lambda}(t) \cdot X(t)\right)\right\}
$$


$=g_{2}^{-1}(t)\left\{D_{2}^{*}(t)\left(P_{\kappa+\lambda}(t) \cdot X(t)\right)-G_{2}(t) D_{1}^{*}(t)\left(P_{\kappa+\lambda}(t) \cdot X(t)\right)\right\}$.

Thereby:

Theorem 1 homogeneous problem (13) solving in $R_{0}$, if $\kappa \geq \mu$, then, its general solution are $\Phi(z)$ and $H(t)$, which are given by (23) and (24) or (25). $P_{\kappa+\lambda}(z)$ is a polynomial vector whose components $P_{k}(z) \quad(k=1, \ldots, n-1)$ are polynomials whose order is no more then $\kappa+\lambda$ and $P_{n}(z)$ is a polynomial whose order is no more then $\kappa-\mu$; if $\kappa<\mu$, then $P_{n}(z)$ must be taken as constant zero and all the other is kept.

Doing the systematical prolongation as (6) after $\Phi(z)$ is obtained, the $\Phi_{*}(z)$ can be obtained easily. Taking $\Phi(z)$ and $\Phi_{*}(z)$ into expression (11), then we get $\Omega(z)$, which is the solution of the problem (9) and (10).

However, the solution (23), (24) or (25) couldn't be used directly because the canonical solution matrix related to the partial indexes. In other words, to get the canonical solution matrix, we must determine the partial indexes at first. It's well known that there's no practical method or formula to calculate them. Hence, (23), (24) or (25) is only a formal solution rather than analytic solution.

Now we directly solve the problem and give the analytic solution from the expression (18).

By the definition of index, we have

$$
\begin{gathered}
\kappa=\frac{1}{2 \pi i}\left[\log \operatorname{det} G_{0}(t)\right]_{L}=\frac{1}{2 \pi}\left[\arg \operatorname{det} G_{0}(t)\right]_{L} . \\
\operatorname{Let} \Lambda(z)=\operatorname{diag}\left(1,1, \cdots, z^{-\kappa}\right)_{n \times n} ; \\
\Lambda^{*}(z)=\operatorname{diag}\left(1,1, \cdots, z^{\kappa}\right)_{n \times n}, \\
G_{e}(t)=\Lambda(t) G_{0}(t),
\end{gathered}
$$

and define the functional value of $G_{e}(t)$ at removable isolated singular point is equal to its limiting value in addition. Then, the original problem is changed to

$$
\Psi^{+}(t)=\Lambda^{*}(t) G_{e}(t) \Psi^{-}(t) .
$$

By introducing function

$$
\begin{aligned}
& \Omega(z)=\left\{\begin{array}{ll}
\Psi(z), & z \in D^{+} \\
\Lambda^{*}(z) \Psi(z), & z \in D^{-}
\end{array},\right. \\
& \Psi(z)=\left\{\begin{array}{l}
P(z) \cdot X_{e}(z) \\
\Lambda(z)\left(P(z) \cdot X_{e}(z)\right)
\end{array}= \begin{cases}\sum_{k=1}^{n} P_{k}(z) X_{e}{ }^{k}(z) & z \in D^{+} \\
\Lambda(z) \sum_{k=1}^{n} P_{k}(z) X_{e}{ }^{k}(z) & z \in D^{-}\end{cases} \right.
\end{aligned}
$$

the problem is reduced to an equivalence problem

$$
\Omega^{+}(t)=G_{e}(t) \Omega^{-}(t) \quad t \in L .
$$

And

$$
\kappa=\frac{1}{2 \pi i}\left[\log \operatorname{det} G_{e}(t)\right]_{L}=\frac{1}{2 \pi}\left[\arg \operatorname{det} G_{e}(t)\right]_{L}=0
$$

\section{Results}

The solving problem of the regular functions Riemann boundary value problem (18) in $R_{\lambda}$ can be transformed to an equivalent regular functions Riemann boundary value problem (30) in $R_{\kappa+\lambda}$, in which the total index of the coefficient matrix $G_{e}(t)$ is equal to zero.

Solving the R-problem (30), we can get the basic solution matrix (seeing refs [1]). The basic solution matrix is the canonical solution matrix of (30). The result has been proven by us in other paper, so we don't repeat it here.

The result mentioned in last paragraph shows that the partial index is an unnecessary conception. Solving regular vector function R-problem, we can get an explicit canonical solution matrix $X(z)$ :

$$
X_{e}^{+}(t)=G_{e}(t) X_{e}^{-}(t), \text { or } G_{e}(t)=X_{e}^{+}(t)\left[X_{e}^{-}(t)\right]^{-1} .
$$

The problem (30) can be written as

$$
\left[X_{e}^{+}(t)\right]^{-1} \Omega^{+}(t)=\left[X_{e}^{-}(t)\right]^{-1} \Omega^{-}(t) .
$$

Let $F(z)=\left[X_{e}(z)\right]^{-1} \Omega(z)$, then it is a holomorphic vector function on whole complex plane. Since the solving process of the equivalent problem is taken in class $R_{\kappa+\lambda}$, Liouville theorem shows that it is only a polynomial vector which denoted by $P(z)$,

$$
P(z)=\left(P_{1}(z), P_{2}(z), \cdots \cdots, P_{n}(z)\right)^{\mathrm{T}},
$$

the order of $P(z)$ is no more than $\kappa+\lambda$.

Taking it into the original problem, we get the expression of the general solution of (16) in $R_{0}$ as

where $X(z)=\left(X^{1}(z), X^{n}(z), \cdots \cdots, X^{n}(z)\right)$, and $P_{k}(z)(k=1, \ldots, n)$ are polynomials whose orders are no more then $\kappa+\lambda$.

Taking $\Psi(z)$ into the express (17), we obtain the $\Phi(z)$ 


$$
\Phi(z)=\left\{\begin{array}{l}
D_{2}^{*}(z) P(z) \cdot X_{e}(z) \\
D_{1}^{*}(z) \Lambda(z)\left(P(z) \cdot X_{e}(z)\right)
\end{array}= \begin{cases}\sum_{k=1}^{n} D_{1 k}^{*}(z) P_{k}(z) X_{e}^{k}(z) & z \in D^{+} \\
\sum_{k=1}^{n} D_{2 k}^{*}(z) \Lambda_{k}(z) P_{k}(z) X_{e}^{k}(z) & z \in D^{-}\end{cases}\right.
$$

$H(t)$ is still given by expression (24) or (25), but the $\Phi(z)$ in it is replaced by (36).

Summarizing the result above, we have

Theorem 2 Irregular vector H-problem (2), (3), (4), (5) solving in $R_{0}$, if $\kappa \geq \mu$, then its general solution is $(\Phi(z), H(t))$, which is given by (36) and (24) or (25). $P(z)$ is a vector whose components $P_{k}(z)(k=1, \ldots, n-1)$ are polynomials whose order is no more then $\mathrm{K}$, and $P_{n}(z)$ is polynomial whose order is no more then; if $\kappa<\mu$, then $P_{n}(z)$ must be taken as 0 and other components $P_{k}(z)(k=1$, $\ldots, n-1)$ are kept.

\section{Discussion and Conclusion}

In the paper, by the method of introducing a special diagonal matrix, we transform the solving problem of irregular set of functions Riemann boundary value inverse problem to a regular Riemann boundary value inverse problem.

The diagonal matrix in our paper here is quite peculiar, in which all its elements are taken as number 1 except the last one. Of course, the last element must take the value that we require. What we need to point out is that this isn't necessary. The user could arrange the element of diagonal matrix in a position according to their own requirement. The only rule one should obey is keep the determinant value fixed. And if the partial index should be introduced, the solvable condition must be written out in details.

Since it has been proved in our past result that the solving process do not have to use the conception of partial index (seeing refs [11]), we omit the relative discussion. In fact, we haven't found any way to calculate it by far.

Though the result comes from solving $G_{0}(t)$ instead of $G(t)$, the difficulty of the solving process the same as well.

\section{Acknowledgements}

This paper is supported by National Natural of Science Foundation (Grand 11201043) of China and Educational Research Funds of the Dalian Maritime University (Grand 2016Y40).

\section{References}

[1] Lu jianke. Boundary Value Problem of Analytic Function [M]. Shanghai: Shanghai science and technology public house, 1987 , 334-388 (in Chinese).

[2] И. I. Muskhelishvili. Singular Integral Equations [M]. Shanghai: Shanghai science and technology public house, 1966 (in Chinese).

[3] F. D. Gakhov. Boundary Value Problem [M] New York: Dover Publication, Inc. New York, 1971.43-290; 400-485.

[4] K. M. Case. Singular integral equations [J], J. Math. Phy. 1966, 7 (12), 2121-2134.

[5] Yang Xiaochun. A class of regular functions inverse problem of Riemann Boundary value problem. [J]. Journal of Ningxia university (Natural of Science), 1996, 17 (1): 5 (in Chinese).

[6] Li Yubo. Irregular Riemann boundary value problem and its applied of the solving singular integral equation with Hilebert kennel (I) [J]. Transaction of Wuhan university (Natural of Science) (in Chinese), 1984, 1:1.

[7] Du Jinyuan. On the trigonometric polynomials interpolating approximate solutions of singular intergral equations with Hilbert kernel. Intergral Equations and Boundary Value Problems [M] (ed. by G. C. Wen, Z. Zhao). Singgapore: World Scientific, 1991, 26-33.

[8] Ding Yun. Non-regular Riemann Boundary Value Problem of Equations, Journal of Ningxia University (natural Science edition), Vol. 28 No. 4, 2007, 305 307 (in Chinese).

[9] Ding Yun, Yang Xiaochun. Non-regular Riemann-Hilbert Boundary Value Problem of Equations, Journal of Dalian Nationalities University, Vol. 10 (5), 2008, 432-434.

[10] I. N. VEKUA. Systems of singular integral equations [M]. Shanghai: Shanghai science and technology public house, 1963 (in Chinese).

[11] Ding Yun, Yang Xiaochun. Research of the Canonical Function Matrix of the Functions of Riemann Boundary Value Problem. Advances in Information and Systems Sciences, Vol. 32009 , 423 429.

[12] Ding Yun, Yang Xiaochun. Vector Function Inverse Riemann Boundary Value Problem and Its Solving [J], British Journal of Mathematics and Computer Science, 12 (3), 1-9, 2016 (Article no.BJMCS. 20274). 